\title{
Ectomycorrhizal fungal communities are dominated by mammalian dispersed truffle-like taxa in north-east Australian woodlands
}

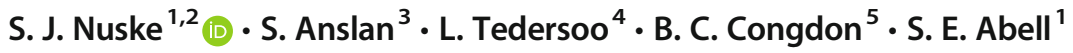

Received: 7 November 2018 / Accepted: 5 March 2019 / Published online: 21 March 2019

(C) The Author(s) 2019

\begin{abstract}
Mycorrhizal fungi are very diverse, including those that produce truffle-like fruiting bodies. Truffle-like fungi are hypogeous and sequestrate (produced below-ground, with an enclosed hymenophore) and rely on animal consumption, mainly by mammals, for spore dispersal. This dependence links mycophagous mammals to mycorrhizal diversity and, assuming truffle-like fungi are important components of mycorrhizal communities, to plant nutrient cycling and ecosystem health. These links are largely untested as currently little is known about mycorrhizal fungal community structure and its dependence on mycophagous mammals. We quantified the mycorrhizal fungal community in the north-east Australian woodland, including the portion interacting with ten species of mycophagous mammals. The study area is core habitat of an endangered fungal specialist marsupial, Bettongia tropica, and as such provides baseline data on mycorrhizal fungi-mammal interactions in an area with no known mammal declines. We examined the mycorrhizal fungi in root and soil samples via high-throughput sequencing and compared the observed taxa to those dispersed by mycophagous mammals at the same locations. We found that the dominant root-associating ectomycorrhizal fungal taxa (>90\% sequence abundance) included the truffle-like taxa Mesophellia, Hysterangium and Chondrogaster. These same taxa were also present in mycophagous mammalian diets, with Mesophellia often dominating. Altogether, $88 \%$ of truffle-like taxa from root samples were shared with the fungal specialist diet and $52 \%$ with diets from generalist mammals. Our data suggest that changes in mammal communities, particularly the loss of fungal specialists, could, over time, induce reductions to truffle-like fungal diversity, causing ectomycorrhizal fungal communities to shift with unknown impacts on plant and ecosystem health.
\end{abstract}

Keywords Sequestrate fungi $\cdot$ Truffle-like fungi $\cdot$ Mycophagy $\cdot$ Ecosystem interactions $\cdot$ Bettongia tropica $\cdot$ Ectomycorrhizal fungi

Electronic supplementary material The online version of this article (https://doi.org/10.1007/s00572-019-00886-2) contains supplementary material, which is available to authorized users.

\section{S. J. Nuske}

susan.nuske@slu.se

1 College of Science and Engineering, Centre for Tropical Environmental and Sustainability Science, Australian Tropical Herbarium, James Cook University, Cairns, QLD 4878, Australia

2 Present address: Department of Forest Ecology and Management, Swedish University of Agricultural Sciences, 90183 Umeå, Sweden

3 Zoological Institute, Braunschweig University of Technology, Mendelssohnstr. 4, 38106 Braunschweig, Germany

4 Natural History Museum and Institute of Ecology and Earth Sciences, University of Tartu, 14A Ravila, EE-50411 Tartu, Estonia

5 College of Science and Engineering, Centre for Tropical Environmental and Sustainability Science, James Cook University, Cairns, QLD 4878, Australia

\section{Introduction}

Hypogeous, sequestrate (truffle-like) mycorrhizal fungi are an important component of forest ecosystems and they rely on animals, particularly mammals, for their spore dispersal (Claridge and May 1994). This implies that truffle-like fungal diversity is likely linked to mammal diversity (Vernes 2007). Disruption to complex ecological networks, such as this mammal-fungi-plant interaction, can cause loss of biodiversity. For example, it is logical to assume that reduced spore dispersal via loss of mammal abundance and diversity would reduce gene flow among truffle-like populations, resulting in undocumented impacts on truffle-like fungal community structure and potential species extinctions. Loss of truffle-like species diversity may in turn alter mycorrhizal communities, potentially impacting fungi-plant interactions. 
Mammals are also thought to play a pivotal role in plantmycorrhiza symbioses, and by extension, plant productivity, diversity and ecosystem health (Maser et al. 1978; Malajczuk et al. 1987; Johnson 1996; Vernes 2007). Such a hypothesis assumes that truffle-like taxa are important components of functioning mycorrhizal communities and that the higher the proportion of truffle-like taxa within the overall mycorrhizal community, either in terms of relative abundance or diversity, the higher the potential influence that mammalian spore dispersal has on the structuring of mycorrhizal and plant communities. Yet, these assumed linkages remain largely untested.

To understand the strength of the relationship between mycorrhizal communities and mycophagous mammals, first we must understand how important truffle-like taxa are to functioning mycorrhizal communities. Few studies have identified the fruiting habits of the various components of the Australian mycorrhizal fungal community (or presented data with enough resolution that this can be inferred post hoc; Online Resource 1, Table S1). In three different studies of ectomycorrhizal (ECM) fungal sporocarps in Australia (Reddell et al. 1999; $\mathrm{Lu}$ et al. 1999; Adams et al. 2006), between 18 and 27\% of taxa found were truffle-like (reported as hypogeous). However, these three surveys are difficult to compare in terms of the richness of hypogeous versus epigeous species because the same methodology was not used for both groups.

It is also important to make the distinction between ECM and arbuscular mycorrhizal (AM) communities as the relative diversity of truffle-like species are quite different in these groups as are their distribution, ecology and interactions with host plants. AM fungi associate with $>80 \%$ of global plant diversity and occur in almost every ecosystem where plants are present, while ECM associate with a much smaller proportion (Brundrett 2009). However, ECM trees can dominate forests in terms of biomass (Reddell et al. 1999). The diversity of truffle-like (sporocarpic) AM fungi is much lower compared to ECM fungi; at least two AM genera contain trufflelike species (Glomus and Acaulospora; Goto and Maia 2005) while thousands of species of ECM truffle-like fungi are within Basidiomycota, Ascomycota and Zygomycota (Bougher and Lebel 2001; Trappe et al. 2009). Therefore, any influence that mammals may have on mycorrhizal communities will likely depend on the differences between these groups.

To our knowledge, only one study has quantified the ECM community on natural forest plant host roots and compared this to mycophagous mammalian diets. Izzo et al. (2005) sampled roots from subtropical North America and found at least $21 \%$ of taxa were truffle-like and between 25 and $40 \%$ of ECM dry root biomass were truffle-like taxa (reported as hypogeous). However, this study was limited to Sanger sequencing of DNA samples from mammalian scats and consequently detected only three truffle-like taxa in mammalian diets. Modern high-throughput sequencing technologies that amplify and sequence DNA from complex communities provide the necessary resolution to examine fungal communities from environmental samples like roots, scats and soil (Lindahl et al. 2013).

Our aim was to address the knowledge gap in the structure and relative proportion of mycorrhizal communities, particularly the truffle-like communities that are interacting with mycophagous mammals. Using high-throughput sequencing (Illumina MiSeq), we compared the mycorrhizal fungal communities (concentrating on ECM fungi) across three sample types including plant roots, soil and scats from mycophagous mammals. We compared our results to reference ITS2 sequences of truffle-like morpho-species collected and characterised from an extensive survey undertaken at one of our sampling sites (Abell-Davis 2008). We collected roots, soil and mammalian scat samples within the habitat of a fungal specialist (the northern bettong; Bettongia tropica) and other mycophagous mammals as this provides a baseline measurement of the interaction between root-associating mycorrhizal fungi and mycophagous mammals where limited known loss of mammal diversity has occurred.

\section{Methods}

\section{Field sampling}

Sampling was carried out on the Lamb Range in North Queensland in Australia at Davies Creek National Park $\left(17^{\circ} 1^{\prime} 23.28^{\prime \prime} \mathrm{S}, 145^{\circ} 34^{\prime} 55.71^{\prime \prime} \mathrm{E}\right.$; elevation $\left.600-730 \mathrm{~m}\right)$ in the late dry season (November to December) in 2014. Additional sampling was carried out at two locations in the early wet season (February to March): Danbulla National Park near Tinaroo Dam $\left(17^{\circ} 9^{\prime} 50.30^{\prime \prime} \mathrm{S}, 145^{\circ} 32^{\prime} 11.56^{\prime \prime} \mathrm{E}\right.$; elevation $630-780 \mathrm{~m})$ and Davies Creek in 2015. Six plots $(12 \times 20 \mathrm{~m})$ at each site were established at least $500 \mathrm{~m}$ apart around the animal trapping grid (as used in Nuske et al. 2018) for the collection of soil and root samples. The dominant and putatively ectomycorrhizal tree species were Eucalyptus crebra, E. tindaliae, E. mediocris, Corymbia intermedia, Allocasuarina littoralis, Al. torulosa and Acacia flavescens.

Soil was collected from 40 cores $(0-10 \mathrm{~cm}$ deep, $5 \mathrm{~cm}$ diameter) per plot. The corers were carefully cleaned with $70 \%$ ethanol between plots. Half of the plot $(6 \times 20 \mathrm{~m})$ was also used to collect putative ECM root-associated taxa by raking the top $10 \mathrm{~cm}$ of soil for 60 person-minutes and collecting fine roots. Because the soil was often too compacted and contained large rocks, we were not able to trace fine roots back to the potential host plants. Instead, we collected all fine root material found. When possible, grass roots were eliminated from individual samples by tracing back to the grass plant. Preliminary work also suggested that a higher volume of roots was collected with this more targeted approach compared to sieving roots from soil cores. A high volume of root tip material was necessary to obtain three DNA extraction subsamples per plot $(3 \times 0.25 \mathrm{~g}$ 
of wet weight root tips, see below). Soil and roots were frozen $\left(-4{ }^{\circ} \mathrm{C}\right)$ within $24 \mathrm{~h}$ of collection and placed into $-20^{\circ} \mathrm{C}$ as soon as possible (up to 4 days).

Fungal diets of mammals were examined by collecting scat samples from trapped individuals. Trapping and animal handling protocols were as outlined in Nuske et al. (2018). Briefly, medium-sized and small mammals trapped for four consecutive nights each, at two locations and two seasons. We identified mammals according to Van Dyck et al. (2013). We marked each individual by either removing a small patch of hair with scissors at the base of the tail or microchipping (B. tropica only; Minichips, Micro Products Australia, Canning Vale, WA or ISO FDX-B Microchips, OzMicrochips, Peakhurst, NSW). Scats were collected from the bottom of Elliot traps or from plastic placed under each cage trap. All traps and plastic were initially cleaned with $70 \%$ ethanol and then re-cleaned if an animal was caught. Scats were stored on ice or in a portable fridge $\left(4^{\circ} \mathrm{C}\right)$ in the field and transferred to $-20^{\circ} \mathrm{C}$ as soon as possible (within 4 days). Each animal was handled according to James Cook University animal ethical guidelines (Approved ethics application A2044).

\section{Laboratory}

Roots were cleaned of excess soil in reverse osmosis water. Fine roots were examined under a dissecting microscope. For each cluster of fine roots collected within a plot, the same volume of root tips of each mycorrhizal morphotype were placed into three subsamples $(0.25 \mathrm{~g})$ for DNA extraction. We did not attempt to verify the colonisation of each ectomycorrhizal morphotype to maintain efficient processing.
Therefore, we consider the mycorrhizal community from root samples to be 'root-associated'. These taxa are more likely to represent functioning mycorrhizal fungi (i.e. those taxa colonised and interacting with plants) compared to that sequenced from soil samples. For soil samples, each of the 40 soil cores per plot were homogenised and pooled. Then three subsamples of fine powdered soil were taken per plot for DNA extraction $(0.25 \mathrm{~g})$. We only used scats from the first capture of an individual per trapping session. Each boluse of scat was broken in half and a small sample of faecal material removed from the inside with sterile forceps. Samples were homogenised and $0.25 \mathrm{~g}$ was taken for DNA extraction.

DNA extraction, PCR, sequencing protocols and bioinformatics were processed as in Nuske et al. (2018). Briefly, DNA was extracted using PowerLyser PowerSoil DNA Isolation kit (Mo Bio, Carlsbad, CA USA) following manufacturer's instructions, except that the samples were lysed using a Qiagen Tissue Lyser for $2 \times 30 \mathrm{~s}$ at $30 \mathrm{~Hz}$, swapping the position of the samples between runs. DNA was amplified with ITS3Mix 1-5 (5'CTAGACTCGTCANCGATGAAGAACGYRG$3^{\prime}$ ) and barcoded ITS4ngs (5'-TCCTSCGCTTATTG ATATGC-3') primers (Tedersoo et al. 2014). The primers were tagged with 10-11 base unique molecular identifiers (MIDs) to later distinguish samples with sequencing runs (Online Resource 1, Table S1). We used negative (for DNA extraction and PCR) and positive controls (PCR) throughout the experiment. Normalised amplicons were subjected to ligation of Illumina adaptors using the TruSeq DNA PCR-free HT Sample Prep kit (Illumina Inc., San Diego, CA, USA). All samples were sequenced using Illumina MiSeq $2 \times 300$ paired-end technology. Raw Illumina data is deposited in Sequence Read Archive (SRA; bioproject SRP150847).
Fig. 1 Rarefied accumulation curve $\pm 95 \%$ confidence intervals for a mycorrhizal OTUs and $\mathbf{b}$ truffle (truffle-like/sequestrate) OTUs. Orange lines are soil samples ( $n=18$, each containing three pooled samples), light blue lines are root samples $(n=18$, each containing three pooled samples) and dark blue lines are scat samples $(n=61)$
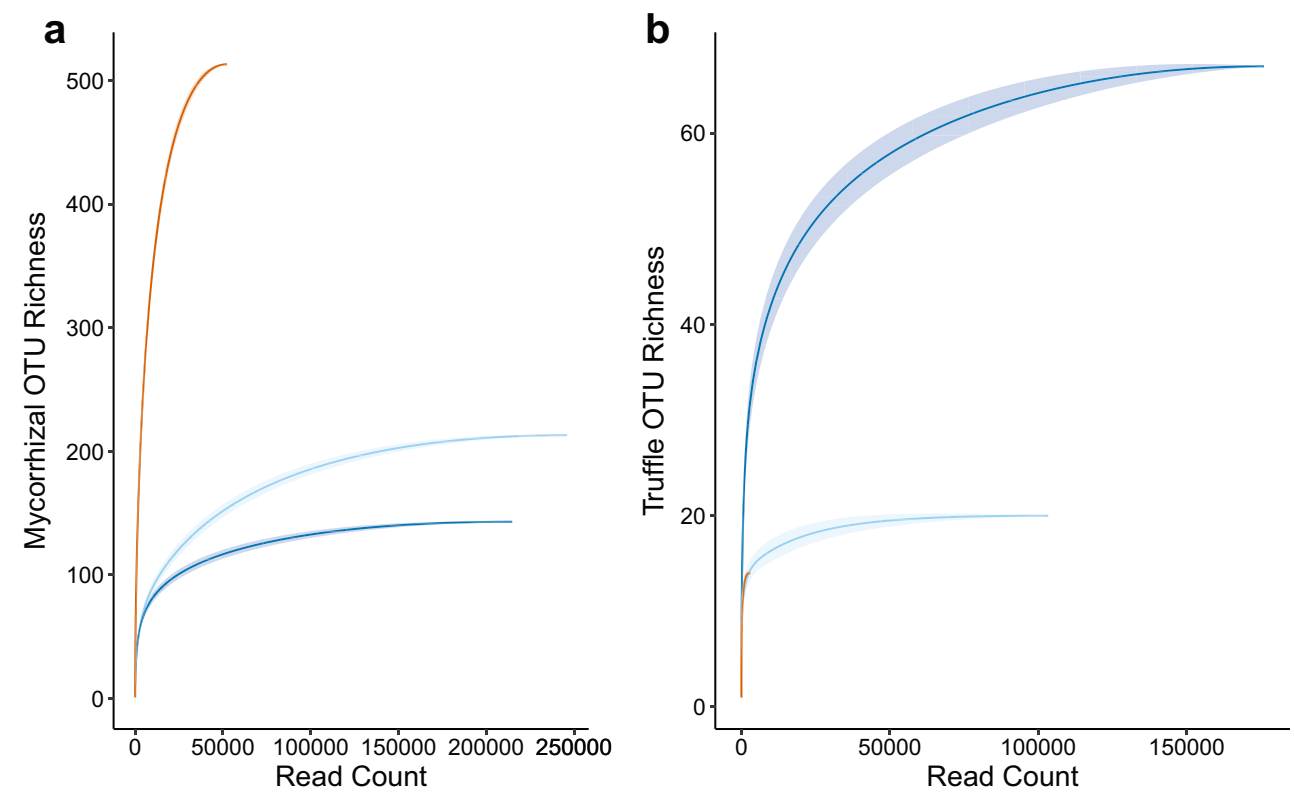
Bioinformatics were performed using PipeCraft (v1.0; Anslan et al. 2017) as per Nuske et al. (2018). Representative sequences for each operational taxonomic unit (OTU, clustered with CD-HIT at 97\% similarity threshold, v4.6; Li and Godzik 2006) were chosen using the mothur 'abundance' method and compared against UNITE (v7.0), GenBank ITS and our local truffle-like fungal database (Nuske et al. 2018) to obtain taxonomic affiliation using BLASTn (Camacho et al. 2009). Taxonomic groups were assigned to functional categories using FUNGuild (v1.0; Nguyen et al. 2015a). All Glomeromycota taxa were assigned as arbuscular mycorrhizal.

\section{Statistics}

OTU subsetting and statistics were performed using the 'phyloseq' package (McMurdie and Holmes 2013) in R (R Core Team 2012). Altogether, six samples were removed from further analyses, because these comprised $<500$ filtered sequences. The fungal dataset was examined at three broad levels: at the whole OTU community level, only examining the mycorrhizal OTUs ( $8.4 \%$ of all taxa) and only examining truffle-like taxa (9.3\% of mycorrhizal taxa). The mycorrhizal subset of the data included only taxa that were assigned as 'highly probable' and 'probable' from the FUNGuild output (Nguyen et al. 2015b). Functional guilds are assigned to ECM status by FUNGuild based on genera, with the exception of Russulaceae. Russulaceae is also one of the most OTU-rich families in this dataset; therefore, when comparing mycorrhizal taxa (e.g. as relative richness of mycorrhizal families), Russulaceae are disproportionally over-represented. To make sure ECM taxa were evenly represented at the genus level, ECM OTUs assigned to family level, Russulaceae, were excluded from analyses (note: ECM OTUs assigned to genus and species level within Russulaceae, for example Russula, were retained). Identified truffle-like taxa are listed in Online Resource 2.

The three subsamples per soil or root sample were pooled computationally by mean sequence abundance per OTU as this likely gives the best estimate of richness (Song et al. 2015). To estimate the accumulation of OTUs per sample, we created rarefaction OTU accumulation curves for each sample type with mycorrhizal and truffle OTU data using the "ggrare" function (richness.R, phyloseq extensions; https:// github.com/mahendra-mariadassou/phyloseqextended with added ggplot2 graphics).

We compared mycorrhizal communities between sample types by tabulating the number of OTUs that were shared and unshared (using 'limma' and 'venneuler' packages in $\mathrm{R})$. We ranked OTUs according to their relative abundance per sample (based on sequence count) and considered the 'dominant' portion of the community to be the highest relative abundance that collectively accounted for $>90 \%$ of the relative abundance. The dominant portion accounted for approximately $25 \%$ of the taxa present.

\section{Results}

\section{Richness}

Soil samples were more OTU-rich than root samples, and both were more OTU-rich than scat samples (6689, 3226 and 2932 OTUs, respectively). There were a total of 9358 filtered OTUs across all samples. Most were not assigned to a functional guild (7508 OTUs) by FUNGuild. Of those that were, most were symbiotrophic (805 OTUs, including 344 ECM OTUs and 428 AM OTUs) followed by saprotrophic (754 OTUs). For mycorrhizal OTUs, at equivalent read count, the OTU richness of scats was $\sim 60 \%$ of the root-associating taxa (Fig. 1a). We detected a higher truffle-like OTU richness in scat samples than in root or soil samples (Fig. 1b).
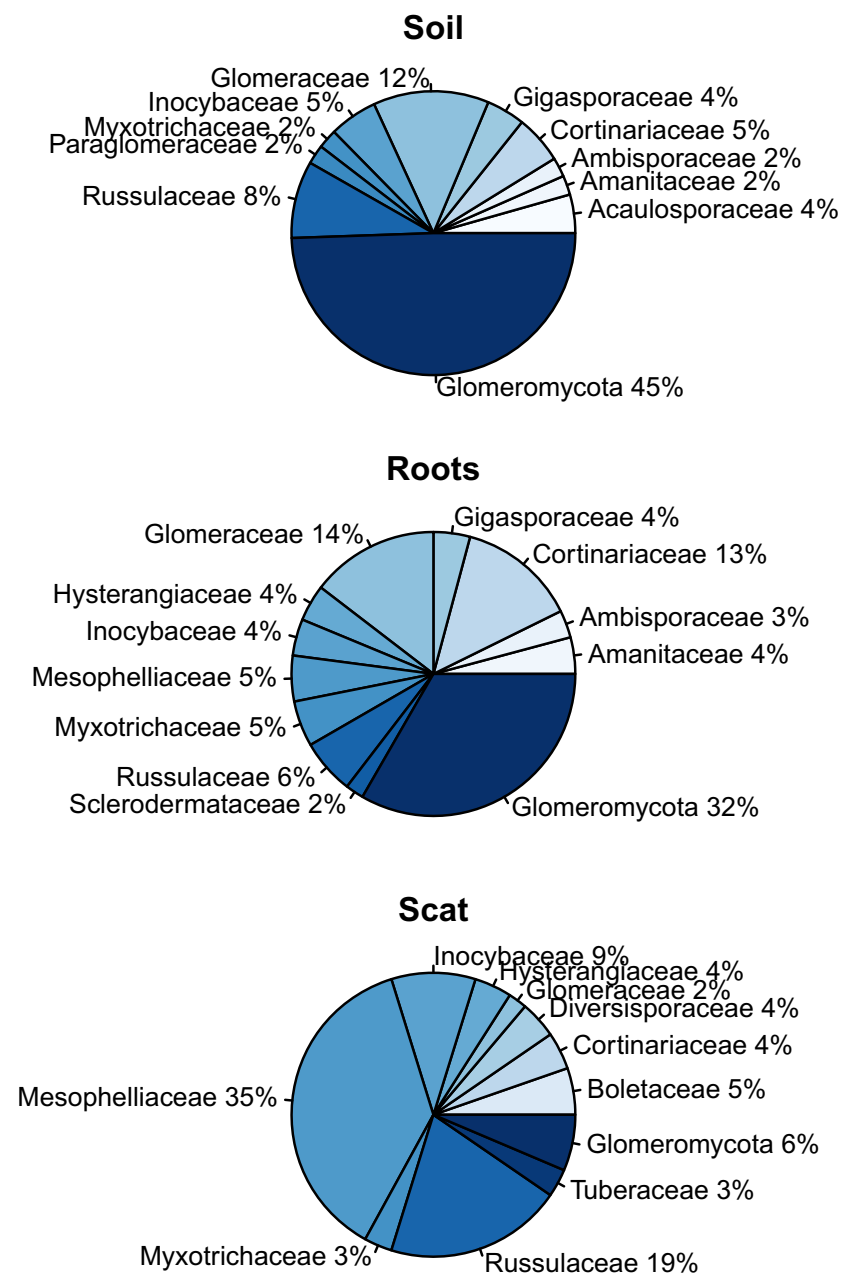

Fig. 2 Relative OTU richness of mycorrhizal families in different samples (soil, roots and scats). Only taxa representing greater than $1 \%$ of the total OTU richness are shown for clarity 
Both soil and root samples had AM fungi (Glomeromycota, Glomeraceae and Gigasporaceae) as the most OTU-rich mycorrhizal taxa. However, they made up < $13 \%$ of the taxa richness in the mammalian scat samples (Fig. 2, including Diversisporaceae) and constituted $<0.01 \%$ of the relative abundance for all samples (Fig. 3). The trufflelike family Mesophelliaceae was the most OTU-rich and highest relative abundance mycorrhizal taxon in mammalian scat samples (Figs. 2 and 3).

Overall, samples had $21.2 \%$ of ECM OTUs as truffle-like taxa (73 OTUs) and $54.1 \%$ as taxa with ambiguous fruiting habit (186 OTUs). Scat samples had a higher proportion of OTUs matching truffle-like fungi than root or soil samples (30-90\%, depending on site and season, compared to 17$23 \%$ for root samples and $7-8 \%$ for soil samples). Scat samples from the fungal specialist (B. tropica) had a higher proportion of ECM truffle-like OTUs (30-90\%) than all other mammal species with generalist diets combined (18-73\%).

\section{Relative abundance}

Truffle-like and secotioid taxa (e.g. Cortinariaceae and Hysterangiaceae) constituted higher proportions of the dominant mycorrhizal root-associating communities compared to soil communities (Table 1; Fig. 3). Soil mycorrhizal communities were dominated by taxa with mixed fruiting habits (e.g. truffle-like taxa and mushroom taxa and Russula and Cortinarius that could represent truffle-like, secotioid or mushroom taxa; Table 1 and Fig. 3). These patterns of relative abundance were consistent across the two sites and two seasons we measured (Table 1). Of the dominant mycorrhizal OTUs associating with root samples, three out of four genera were truffle-like from Hysterangiales (Hysterangium, Mesophellia and Chondrogaster; Table 1). Four truffle-like OTUs were shared between dominant root taxa and mammalian diets and five dominant ECM OTUs were shared between soil and scat samples (Table 1). Within taxa, comparisons showed that Cortinarius was more OTU-rich and more relatively abundant in roots and soil compared to scat samples, whereas Malajczukia and Mesophellia (Mesophelliaceae) were more OTU-rich and abundant in scat samples compared to soil and roots (Table 1; Fig. 2). Hysterangium (Hysterangiaceae) and Chondrogaster (Mesophelliaceae) were relatively more abundant in roots.

\section{Shared taxa between samples}

The percentage of shared taxa between fungal specialist diets and ECM communities on roots (36.6\%) and in soil (28\%) was slightly higher than that for fungal generalist diets (26 and $14.7 \%$, respectively; Fig. 4). The percentage of shared trufflelike OTUs from roots and soil and fungal specialist diets was much higher (87.5 and $78.8 \%$, respectively; Fig. 4). In contrast, just over half of the truffle-like taxa from root and soil samples overlapped with fungal generalist diets $(52-53 \%)$. Arbuscular mycorrhizal (AM) OTU richness was highest from soil samples and AM communities from roots did not overlap significantly with mammalian scat samples (<1.8\%; Fig. 4).
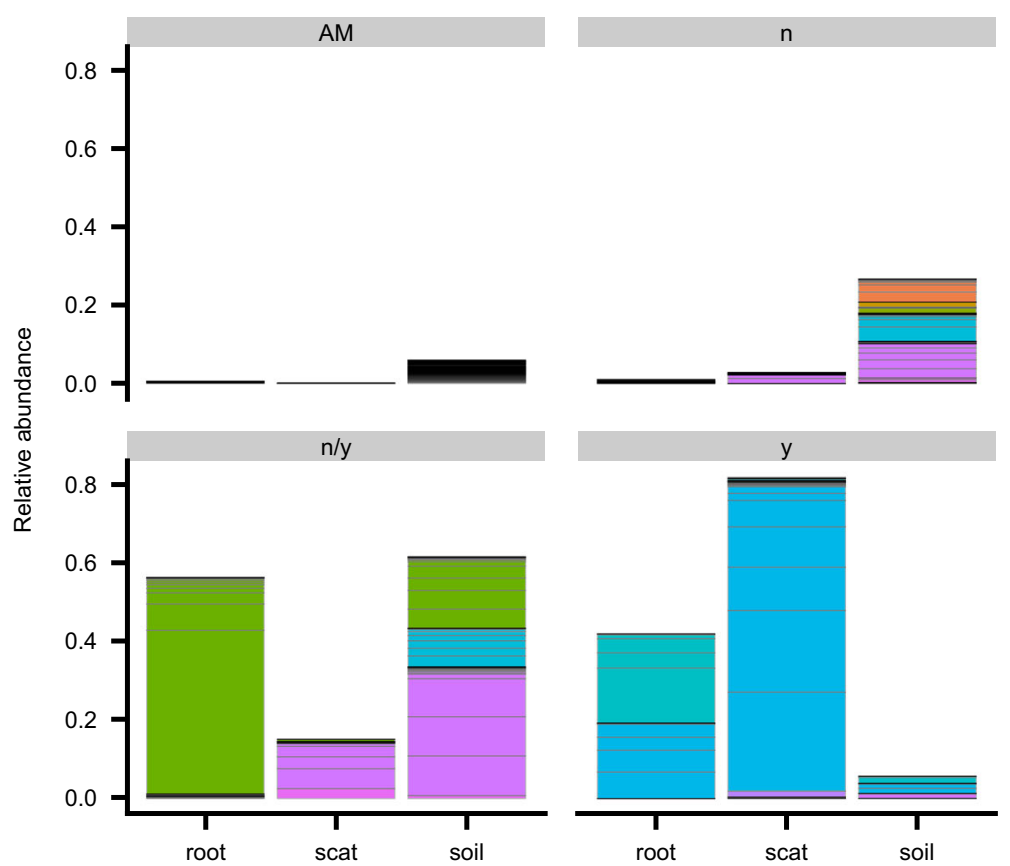

\section{Mycorrhizal Family}

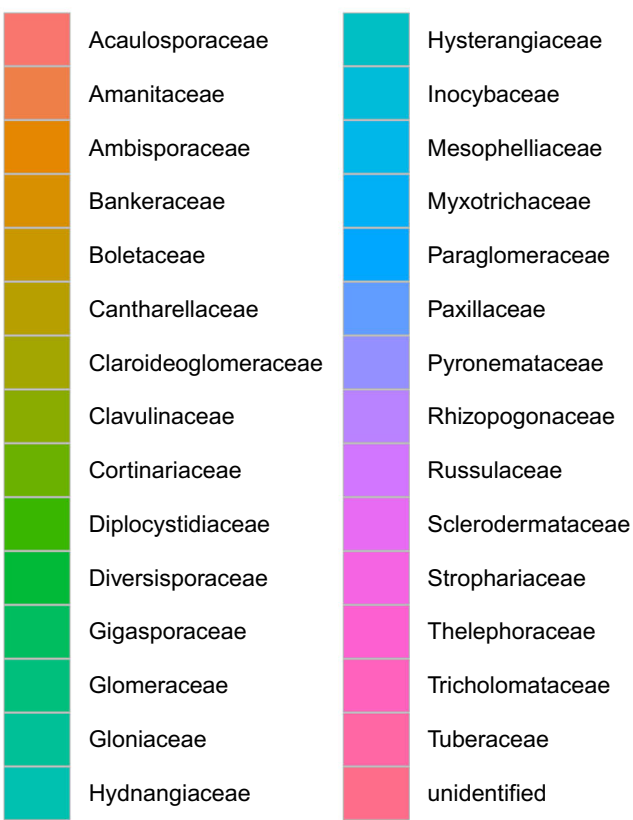

Fig. 3 Relative abundance of mycorrhizal families in different samples (soil, roots and scats) split by mycorrhizal type ( $\mathrm{AM}=$ arbuscular mycorrhizal) and fruiting habit ( $n=$ fruiting habit other than truffle-like taxa, $\mathrm{n} / \mathrm{y}=$ unknown fruiting habit, $\mathrm{y}=$ truffle-like/sequestrate taxa). Black bars are overlapping boundary lines representing OTUs with a relative abundance too small to display 
Table 1 Mycorrhizal OTUs that make up the dominant proportion (cumulatively $90 \%$ of sequence abundance) of samples per site (DC $=$ Davies Creek, TD = Tinaroo Dam), sample type and season, with number of replicates $(n)$, total OTUs per sample group (total), percent relative abundance (RA), accession number, e-value, percentage similarity with database sequence (ID), percentage overlap with reference sequence (Cov) and OTU sequence length (SL). Taxa in boldface are truffle-like/ sequestrate and underlined are secotioid or higher taxa that include truffle-like/sequestrate or secotioid taxa. When fruiting habit is listed at genus level, it applies to the whole genus. Mycorrhizal status (Myc) is either ectomycorrhizal (ECM), ericoid mycorrhizal (ErM) or arbuscular mycorrhizal (AM). The mammalian specialist scats are from Bettongia tropica. The mammalian generalist scats are from Isoodon macrourus, I. obesulus, Melomys sp., Trichosurus vulpecula, Uromys caudimaculatus and Zyzomys argurus

\begin{tabular}{|c|c|c|c|c|c|c|c|c|c|c|c|c|}
\hline Site & Sample & Season & $\mathrm{n}$ & Total & $\mathrm{RA}$ & Fungal taxa & Myc & Accession & e-value & ID & Cov & $\mathrm{SL}$ \\
\hline \multirow[t]{10}{*}{ DC } & \multirow[t]{4}{*}{ roots } & \multirow[t]{4}{*}{ dry } & \multirow[t]{4}{*}{$6^{\mathrm{b}}$} & \multirow[t]{4}{*}{84} & 64.4 & Hysterangium aggregatum & ECM & KY697566-7 & $2.21 \mathrm{E}-133$ & 100 & 97.9 & 333 \\
\hline & & & & & 17.2 & $\underline{\text { Cortinarius globuliformis }}$ & $\mathrm{ECM}$ & AF325582 & $2.50 \mathrm{E}-141$ & 99 & 100 & 350 \\
\hline & & & & & 5.7 & Hysterangium aggregatum & $\mathrm{ECM}$ & KY697566-7 & $2.16 \mathrm{E}-129$ & 98 & 97.9 & 335 \\
\hline & & & & & 5.3 & $\underline{\text { Cortinarius globuliformis }}$ & $\mathrm{ECM}$ & AF325582 & $3.24 \mathrm{E}-142$ & 99 & 100 & 349 \\
\hline & \multirow[t]{6}{*}{ roots } & \multirow[t]{6}{*}{ wet } & \multirow[t]{6}{*}{$6^{\mathrm{b}}$} & \multirow[t]{6}{*}{102} & 44.1 & Cortinarius & ECM & FR731477 & $1.53 \mathrm{E}-143$ & 100 & 99.7 & 350 \\
\hline & & & & & 12.5 & Hysterangium $^{c}$ & ECM & KC222660 & $1.00 \mathrm{E}-144$ & 94 & 100 & 340 \\
\hline & & & & & 11.8 & Hysterangium ef gardneri ${ }^{a}$ & ECM & KY697590 & $3.96 \mathrm{E}-139$ & 100 & 99.7 & 340 \\
\hline & & & & & 11.4 & Hysterangium aggregatum & $\mathrm{ECM}$ & KY697566-7 & $2.21 \mathrm{E}-133$ & 100 & 97.9 & 333 \\
\hline & & & & & 7.1 & Mesophellia oleifera $^{c}$ & ECM & KY697602-3 & $4.15 \mathrm{E}-177$ & 100 & 100 & 425 \\
\hline & & & & & 3.9 & $\underline{\text { Cortinarius }}$ & ECM & KJ421051 & $4.44 \mathrm{E}-112$ & 91 & 100 & 358 \\
\hline \multirow[t]{5}{*}{$\mathrm{TD}$} & \multirow[t]{5}{*}{ roots } & \multirow[t]{5}{*}{ wet } & \multirow[t]{5}{*}{$6^{\mathrm{b}}$} & \multirow[t]{5}{*}{99} & 53.1 & Cortinarius & ECM & FR731477 & $1.53 \mathrm{E}-143$ & 100 & 99.7 & 350 \\
\hline & & & & & 12.5 & Mesophellia ${ }^{c}$ & $\mathrm{ECM}$ & GQ981511 & $2.00 \mathrm{E}-111$ & 91 & 98 & 298 \\
\hline & & & & & 12.4 & Cortinarius & ECM & KF732610 & $5.34 \mathrm{E}-105$ & 89 & 100 & 353 \\
\hline & & & & & 9.3 & Mesophellia oleifera ${ }^{c}$ & ECM & KY697602-3 & $4.86 \mathrm{E}-166$ & 97 & 100 & 420 \\
\hline & & & & & 4.4 & Chondrogaster $s p B / s p F^{a}$ & $\mathrm{ECM}$ & KY697582-5 & $4.75 \mathrm{E}-151$ & 100 & 100 & 366 \\
\hline \multirow[t]{9}{*}{$\mathrm{DC}$} & \multirow[t]{5}{*}{ specialist diet } & \multirow[t]{5}{*}{ dry } & \multirow[t]{5}{*}{7} & \multirow[t]{5}{*}{51} & 31.0 & Mesophellia ${ }^{c, d}$ & $\mathrm{ECM}$ & GQ981511 & $2.00 \mathrm{E}-111$ & 91 & 98 & 298 \\
\hline & & & & & 19.5 & Malajcukia ingrattissima $^{d}$ & $\mathrm{ECM}$ & KY697598 & $6.60 \mathrm{E}-156$ & 100 & 100 & 377 \\
\hline & & & & & 19.3 & Mesophellia oleifera ${ }^{c}$ & $\mathrm{ECM}$ & KY697602-3 & $4.15 \mathrm{E}-177$ & 100 & 100 & 425 \\
\hline & & & & & 17.1 & Mesophellia glauca & ECM & GQ981510 & $9.13 \mathrm{E}-147$ & 98 & 99 & 376 \\
\hline & & & & & 5.8 & Mesophellia glauca & ECM & GQ981511 & $1.00 \mathrm{E}-162$ & 97 & 99 & 354 \\
\hline & \multirow[t]{4}{*}{ specialist diet } & \multirow[t]{4}{*}{ wet } & 6 & 24 & 34.7 & Russula $^{d}$ & ECM & LC006943 & $3.99 \mathrm{E}-138$ & 91 & 100 & 426 \\
\hline & & & & & 32.9 & Mesophellia ${ }^{c, d}$ & ECM & GQ981511 & $2.00 \mathrm{E}-111$ & 91 & 98 & 298 \\
\hline & & & & & 17.5 & $\underline{\text { Russula }}$ & ECM & UDB016041 & $6.92 \mathrm{E}-129$ & 93 & 100 & 376 \\
\hline & & & & & 7.0 & $\underline{\text { Cortinarius }}$ & $\mathrm{ECM}$ & FJ157098 & $8.08 \mathrm{E}-118$ & 92 & 100 & 371 \\
\hline TD & specialist diet & wet & 16 & 70 & 26.4 & Malajcukia ingrattissima $^{d}$ & ECM & KY697598 & $6.60 \mathrm{E}-156$ & 100 & 100 & 377 \\
\hline & & & & & 16.6 & Mesophellia $^{c, d}$ & ECM & GQ981511 & $2.00 \mathrm{E}-111$ & 91 & 98 & 298 \\
\hline & & & & & 15.2 & Mesophellia & $\mathrm{ECM}$ & GQ981511 & $2.00 \mathrm{E}-110$ & 91 & 99 & 295 \\
\hline & & & & & 12.8 & Mesophellia glauca & $\mathrm{ECM}$ & GQ981510 & $9.13 \mathrm{E}-147$ & 98 & 99 & 376 \\
\hline & & & & & 7.2 & $\underline{\text { Russula }}^{d}$ & $\mathrm{ECM}$ & LC008293 & $3.95 \mathrm{E}-138$ & 91 & 100 & 422 \\
\hline & & & & & 6.1 & Scleroderma $\mathrm{spB} / \mathrm{sp} \mathrm{C}^{a, d}$ & $\mathrm{ECM}$ & KY697606-7 & $3.41 \mathrm{E}-146$ & 100 & 100 & 35 \\
\hline & & & & & 3.4 & Mesophellia oleifera $^{c}$ & $\mathrm{ECM}$ & KY697602-3 & $4.86 \mathrm{E}-166$ & 97 & 100 & 420 \\
\hline & & & & & 3.0 & Mesophellia oleifera $^{c}$ & $\mathrm{ECM}$ & KY697602-3 & $4.15 \mathrm{E}-177$ & 100 & 100 & 425 \\
\hline $\mathrm{DC}$ & generalist diets & dry & 11 & 27 & 68.8 & Malajcukia ingrattissima $^{d}$ & $\mathrm{ECM}$ & KY697598 & $6.60 \mathrm{E}-156$ & 100 & 100 & 377 \\
\hline & & & & & 18.7 & Mesophellia ${ }^{c, d}$ & $\mathrm{ECM}$ & GQ981511 & $2.00 \mathrm{E}-111$ & 91 & 98 & 298 \\
\hline & & & & & 9.6 & Mesophellia & $\mathrm{ECM}$ & GQ981511 & $2.00 \mathrm{E}-110$ & 91 & 99 & 295 \\
\hline & generalist diets & wet & 8 & 29 & 35.3 & Mesophellia & $\mathrm{ECM}$ & GQ981511 & $2.00 \mathrm{E}-110$ & 91 & 99 & 29 \\
\hline & & & & & 30.0 & $\underline{\text { Russula }}^{d}$ & $\mathrm{ECM}$ & LC006943 & $3.99 \mathrm{E}-138$ & 91 & 100 & 426 \\
\hline & & & & & 16.8 & $\underline{\text { Russula }}$ & ECM & UDB016041 & $6.92 \mathrm{E}-129$ & 93 & 100 & 376 \\
\hline & & & & & 11.5 & Lactarius rufus & ECM & KT165272 & $4.86 \mathrm{E}-170$ & 100 & 100 & 409 \\
\hline TD & generalist diets & wet & 8 & 24 & 71.4 & Rhizopogon pseudoroseolus & $\mathrm{ECM}$ & AJ810040 & $2.84 \mathrm{E}-168$ & 100 & 100 & 40 \\
\hline & & & & & 9.2 & Mesophellia ${ }^{c, d}$ & ECM & GQ981511 & $2.00 \mathrm{E}-111$ & 91 & 98 & 298 \\
\hline & & & & & 7.9 & Malajcukia ingrattissima $^{d}$ & ECM & KY697598 & $6.60 \mathrm{E}-156$ & 100 & 100 & 377 \\
\hline & & & & & 2.6 & Hysterangium $^{c}$ & $\mathrm{ECM}$ & KC222660 & $1.00 \mathrm{E}-144$ & 94 & 100 & 34 \\
\hline
\end{tabular}


Table 1 (continued)

\begin{tabular}{|c|c|c|c|c|c|c|c|c|c|c|c|c|}
\hline Site & Sample & Season & $\mathrm{n}$ & Total & RA & Fungal taxa & Myc & Accession & e-value & ID & $\mathrm{Cov}$ & SL \\
\hline \multirow[t]{49}{*}{$\mathrm{DC}$} & \multirow[t]{19}{*}{ soil } & \multirow[t]{19}{*}{ dry } & \multirow[t]{19}{*}{$6^{\mathrm{b}}$} & \multirow[t]{19}{*}{196} & 22.4 & Russula & ECM & UDB016041 & $1.08 \mathrm{E}-122$ & 92 & 100 & 370 \\
\hline & & & & & 19.9 & Russula $^{d}$ & ECM & LC006943 & $3.99 \mathrm{E}-138$ & 91 & 100 & 426 \\
\hline & & & & & 11.5 & Russula & ECM & UDB016041 & $4.05 \mathrm{E}-127$ & 92 & 100 & 373 \\
\hline & & & & & 6.4 & Russula anthracina & ECM & UDB011194 & $5.00 \mathrm{E}-151$ & 97 & 100 & 382 \\
\hline & & & & & 4.9 & Amanita & ECM & KP071067 & $2.94 \mathrm{E}-126$ & 94 & 100 & 356 \\
\hline & & & & & 3.6 & Inocybe & ECM & FJ904133 & $1.39 \mathrm{E}-100$ & 88 & 100 & 344 \\
\hline & & & & & 3.5 & Hysterangium aggregatum & ECM & KY697566-7 & $2.21 \mathrm{E}-133$ & 100 & 97.9 & 333 \\
\hline & & & & & 3.1 & $\underline{\text { Russula }}$ & ECM & EU019930.1 & $1.63 \mathrm{E}-104$ & 93 & 80.4 & 382 \\
\hline & & & & & 2.7 & Inocybe & ECM & JQ085932 & $1.01 \mathrm{E}-91$ & 87 & 100 & 346 \\
\hline & & & & & 2.3 & $\overline{\text { Mesophellia }^{d}}$ & ECM & GQ981511 & $2.00 \mathrm{E}-111$ & 91 & 98 & 298 \\
\hline & & & & & 2.1 & Lactarius & ECM & HQ318282 & $2.43 \mathrm{E}-129$ & 94 & 100 & 368 \\
\hline & & & & & 1.3 & Inocybe & ECM & JX178624 & $2.40 \mathrm{E}-98$ & 86 & 100 & 359 \\
\hline & & & & & 1.3 & Auritella serpentinocystis & ECM & KJ729858 & $3.63 \mathrm{E}-150$ & 100 & 100 & 364 \\
\hline & & & & & 1.2 & Cortinarius globuliformis & ECM & AF325582 & $2.50 \mathrm{E}-141$ & 99 & 100 & 350 \\
\hline & & & & & 0.9 & Russula & ECM & UDB016041 & $2.40 \mathrm{E}-125$ & 92 & 100 & 373 \\
\hline & & & & & 0.8 & Hysterangium aggregatum & ECM & KY697566-7 & $2.60 \mathrm{E}-122$ & 97 & 97.9 & 330 \\
\hline & & & & & 0.8 & Russula & ECM & AB509981 & $3.49 \mathrm{E}-138$ & 94 & 99.7 & 380 \\
\hline & & & & & 0.7 & $\overline{\text { Cortinarius }}$ & ECM & KR011131 & $2.92 \mathrm{E}-122$ & 93 & 100 & 363 \\
\hline & & & & & 0.6 & Cortinarius globuliformis & ECM & AF325582 & $3.24 \mathrm{E}-142$ & 99 & 100 & 349 \\
\hline & \multirow[t]{30}{*}{ soil } & \multirow[t]{30}{*}{ wet } & \multirow[t]{30}{*}{$6^{\mathrm{b}}$} & \multirow[t]{30}{*}{251} & 24.8 & Russula & ECM & UDB016041 & $4.05 \mathrm{E}-127$ & 92 & 100 & 373 \\
\hline & & & & & 13.7 & Cortinarius & ECM & GU233352 & $1.41 \mathrm{E}-96$ & 88 & 100 & 357 \\
\hline & & & & & 9.6 & Russula $^{d}$ & ECM & LC006943 & $3.99 \mathrm{E}-138$ & 91 & 100 & 426 \\
\hline & & & & & 8.0 & Auritella chamaecephala & ECM & KT378201 & $9.00 \mathrm{E}-138$ & 97 & 100 & 358 \\
\hline & & & & & 7.5 & Inocybe & ECM & JQ085932 & $1.01 \mathrm{E}-91$ & 87 & 100 & 346 \\
\hline & & & & & 4.7 & Cortinarius & ECM & KJ421051 & $4.44 \mathrm{E}-112$ & 91 & 100 & 358 \\
\hline & & & & & 4.5 & Malajcukia ingrattissima $^{d}$ & ECM & KY697598 & $6.60 \mathrm{E}-156$ & 100 & 100 & 377 \\
\hline & & & & & 2.5 & $\underline{\text { Russula }}$ & ECM & UDB016041 & $1.08 \mathrm{E}-122$ & 92 & 100 & 370 \\
\hline & & & & & 2.3 & Lactarius & ECM & HQ318282 & $2.43 \mathrm{E}-129$ & 94 & 100 & 368 \\
\hline & & & & & 1.8 & Mesophellia ${ }^{d}$ & ECM & GQ981511 & $2.00 \mathrm{E}-111$ & 91 & 98 & 298 \\
\hline & & & & & 1.5 & Amanita & ECM & JF899547 & $4.64 \mathrm{E}-112$ & 89 & 100 & 371 \\
\hline & & & & & 1.1 & Cantharellus & ECM & AB509732 & 8.03E-106 & 85 & 99.7 & 398 \\
\hline & & & & & 0.9 & $\underline{\text { Russula }}$ & ECM & UDB016041 & $3.12 \mathrm{E}-126$ & 92 & 100 & 373 \\
\hline & & & & & 0.8 & Auritella serpentinocystis & ECM & KJ729858 & $3.63 \mathrm{E}-150$ & 100 & 100 & 364 \\
\hline & & & & & 0.7 & Russula & ECM & KM373243 & $3.51 \mathrm{E}-134$ & 93 & 100 & 391 \\
\hline & & & & & 0.7 & Hysterangium aggregatum & ECM & KY697566-7 & $2.21 \mathrm{E}-133$ & 100 & 97.9 & 333 \\
\hline & & & & & 0.6 & Scleroderma $s p B / s p C^{a, d}$ & ECM & KY697606-7 & $3.41 \mathrm{E}-146$ & 100 & 100 & 355 \\
\hline & & & & & 0.5 & Oidiodendron & $\mathrm{ErM}^{\mathrm{e}}$ & AF062808.1 & $1.51 \mathrm{E}-105$ & 95 & 100 & 291 \\
\hline & & & & & 0.5 & Glomeromycetes & $\mathrm{AM}$ & JF276264 & $4.84 \mathrm{E}-128$ & 96 & 100 & 348 \\
\hline & & & & & 0.4 & Auritella & ECM & KT378201 & $4.51 \mathrm{E}-116$ & 92 & 100 & 354 \\
\hline & & & & & 0.4 & Glomerales & $\mathrm{AM}$ & AY394681 & $3.63 \mathrm{E}-76$ & 81 & 100 & 380 \\
\hline & & & & & 0.3 & Glomerales & $\mathrm{AM}$ & HE794042 & $2.00 \mathrm{E}-117$ & 93 & 100 & 338 \\
\hline & & & & & 0.3 & Glomeraceae & $\mathrm{AM}$ & KM226647 & $1.20 \mathrm{E}-115$ & 97 & 88.6 & 343 \\
\hline & & & & & 0.3 & Inocybe & ECM & JX178624 & $2.40 \mathrm{E}-98$ & 86 & 100 & 359 \\
\hline & & & & & 0.3 & Glomerales & $\mathrm{AM}$ & KP235575 & $1.87 \mathrm{E}-101$ & 90 & 97.5 & 354 \\
\hline & & & & & 0.3 & Oidiodendron & $\mathrm{ErM}^{\mathrm{e}}$ & KX640607 & $3.00 \mathrm{E}-131$ & 96 & 99.7 & 289 \\
\hline & & & & & 0.2 & Russula & ECM & UDB016041 & $8.38 \mathrm{E}-122$ & 91 & 99.5 & 373 \\
\hline & & & & & 0.2 & Glomerales & $\mathrm{AM}$ & JX276895 & $4.56 \mathrm{E}-124$ & 95 & 100 & 340 \\
\hline & & & & & 0.2 & Glomerales & $\mathrm{AM}$ & KM226647 & $3.30 \mathrm{E}-80$ & 86 & 88.6 & 343 \\
\hline & & & & & 0.2 & Glomerales & $\mathrm{AM}$ & AY394681 & $1.72 \mathrm{E}-81$ & 82 & 100 & 374 \\
\hline
\end{tabular}


Table 1 (continued)

\begin{tabular}{|c|c|c|c|c|c|c|c|c|c|c|c|c|}
\hline Site & Sample & Season & $\mathrm{n}$ & Total & $\mathrm{RA}$ & Fungal taxa & Myc & Accession & e-value & ID & $\mathrm{Cov}$ & SL \\
\hline \multirow[t]{26}{*}{$\mathrm{TD}$} & \multirow[t]{26}{*}{ soil } & \multirow[t]{26}{*}{ wet } & \multirow[t]{26}{*}{$6^{\mathrm{b}}$} & \multirow[t]{26}{*}{289} & 12.2 & Cortinarius globuliformis & ECM & AF325582 & $6.96 \mathrm{E}-141$ & 99 & 100 & 351 \\
\hline & & & & & 11.5 & Cortinarius globuliformis & ECM & AF325582 & $3.24 \mathrm{E}-142$ & 99 & 100 & 349 \\
\hline & & & & & 9.2 & Inocybe alienospora & ECM & KP171105 & $1.88 \mathrm{E}-140$ & 99 & 100 & 343 \\
\hline & & & & & 6.9 & $\underline{\text { Cortinarius }}$ & ECM & FR731477 & $1.53 \mathrm{E}-143$ & 100 & 99.7 & 350 \\
\hline & & & & & 6.5 & $\overline{\text { Amanita egregia }}$ & ECM & KP012748 & $2.82 \mathrm{E}-134$ & 100 & 100 & 328 \\
\hline & & & & & 5.6 & Lactarius & ECM & AB509713 & $4.60 \mathrm{E}-112$ & 90 & 99.7 & 369 \\
\hline & & & & & 5.0 & Inocybe & ECM & KP308804 & $6.00 \mathrm{E}-135$ & 89 & 94 & 359 \\
\hline & & & & & 4.8 & Inocybe & $\mathrm{ECM}$ & KP308804 & $3.05 \mathrm{E}-99$ & 87 & 100 & 352 \\
\hline & & & & & 4.4 & Lactarius eucalypti & ECM & UDB002671 & $1.70 \mathrm{E}-162$ & 96 & 100 & 420 \\
\hline & & & & & 3.2 & Clavulina & $\mathrm{ECM}$ & JQ724058 & $3.49 \mathrm{E}-103$ & 85 & 100 & 383 \\
\hline & & & & & 3.1 & Austroboletus subvirens & $\mathrm{ECM}$ & KP242209 & $5.04 \mathrm{E}-155$ & 100 & 100 & 375 \\
\hline & & & & & 2.8 & Zelleromyces spE & ECM & KY697617-9 & $8.92 \mathrm{E}-153$ & 96 & 100 & 399 \\
\hline & & & & & 2.6 & Lactifluus & $\mathrm{ECM}$ & KM282287 & $1.36 \mathrm{E}-127$ & 95 & 100 & 351 \\
\hline & & & & & 2.1 & Inocybe & $\mathrm{ECM}$ & AM882711 & 2.72E-99 & 90 & 100 & 321 \\
\hline & & & & & 2.0 & $\underline{\text { Russula }}$ & $\mathrm{ECM}$ & UDB016041 & $1.08 \mathrm{E}-122$ & 92 & 100 & 370 \\
\hline & & & & & 1.5 & ${\underline{\text { Scleroderma } s p B / s p C^{a, d}}}^{-}$ & $\mathrm{ECM}$ & KY697606-7 & $3.41 \mathrm{E}-146$ & 100 & 100 & 355 \\
\hline & & & & & 1.4 & $\underline{\text { Russula }}^{d}$ & $\mathrm{ECM}$ & LC006943 & $3.99 \mathrm{E}-138$ & 91 & 100 & 426 \\
\hline & & & & & 1.1 & Pisolithus croceorrhizus & ECM & JN847473 & $8.64 \mathrm{E}-157$ & 100 & 100 & 379 \\
\hline & & & & & 0.8 & Inocybe violaceocaulis & ECM & КР641643 & $4.75 \mathrm{E}-151$ & 100 & 100 & 366 \\
\hline & & & & & 0.8 & Amanita & ECM & GU222312 & $3.39 \mathrm{E}-111$ & 92 & 94.1 & 356 \\
\hline & & & & & 0.6 & Pisolithus croceorrhizus & $\mathrm{ECM}$ & JN847473 & $6.64 \mathrm{E}-156$ & 99 & 100 & 379 \\
\hline & & & & & 0.5 & $\underline{\text { Cortinarius globuliformis }}$ & ECM & AF325582 & $3.25 \mathrm{E}-142$ & 99 & 100 & 350 \\
\hline & & & & & 0.5 & Amanita & $\mathrm{ECM}$ & $\mathrm{AB} 015702$ & $8.29 \mathrm{E}-95$ & 87 & 100 & 355 \\
\hline & & & & & 0.5 & Glomerales & $\mathrm{AM}$ & JN195694 & $5.02 \mathrm{E}-132$ & 96 & 100 & 350 \\
\hline & & & & & 0.5 & Mesophellia & $\mathrm{ECM}$ & GQ981511 & $8.00 \mathrm{E}-116$ & 92 & 97 & 301 \\
\hline & & & & & 0.4 & $\underline{\text { Russula }}^{d}$ & ECM & LC008293 & $3.95 \mathrm{E}-138$ & 91 & 100 & 422 \\
\hline
\end{tabular}

${ }^{a}$ Indicates taxa that are indistinguishable from ITS2 sequences (within 3\% similarity) from morphological groups identified in Abell-Davis (2008), Online Resource 2

${ }^{\mathrm{b}}$ Includes three subsamples per sample pooled computationally

${ }^{\mathrm{c}}$ Indicates OTUs that are shared between root and scat samples

${ }^{\mathrm{d}}$ Indicates OTUs that are shared between scat and soil samples

${ }^{\mathrm{e}}$ The ericaceous shrub, Melichrus urceolatus, was present at low abundance at this site

Almost all Hysterangiaceae, Mesophelliaceae and Tuberaceae truffle and truffle-like taxa sequenced from roots and soil were also recovered from mammalian scats (Fig. 5). Hysterangiaceae and Mesophelliaceae made up between 28 and $71 \%$ of the ECM sequence abundance of root samples (depending on site and season; Table 1). Of the families sequenced from root samples, over half (59\%) of Russulaceae, $20 \%$ of Inocybaceae and $7 \%$ of Cortinariaceae were also in scat samples (Fig. 5).

\section{Discussion}

The hypothesis that mammal communities are important for plant-mycorrhizal relationships, and indirectly are also contributing to the health of mycorrhizal host trees and nutrient cycling (Johnson 1996) assumes that mammal-dispersed truffle-like fungi are an important part of mycorrhizal communities. Our data support this assumption, at least in north-east Australian woodlands with a diverse mammalian community including the fungal specialist, B. tropica. Dominant components of the root-associating mycorrhizal community were ECM truffle-like taxa dispersed by mammals. In another study of tropical ECM communities, truffle-like taxa (Hysterangiales; Hysterangium and Nothocastoreum) were also found in the dominant portion as sporocarps and on roots (Reddell et al. 1999). This indicates that mammals can potentially have a substantial influence on the functioning ECM community. Additionally, the fungal specialist, B. tropica, has previously found to consume a higher diversity and more unique truffle-like taxa than the combined diets of fungal generalists in the same community (Nuske et al. 2018). Indeed, 
most truffle-like taxa associating with roots were within the fungal specialists' diet. By dispersing a high diversity of ECM inocula, these mammals, particularly fungal specialists, appear to indirectly contribute to plant productivity and nutrient cycling in these ecosystems.

The hypothesis linking mycophagous mammals to plant health and ecosystem functioning also implies that if the mammal community were to be altered, then the inoculum available for new colonising roots is also altered (over time, potentially lowering truffle-like fungal diversity). If dispersal of these taxa were reduced via changes to the mammal community, then the ECM community is likely to experience major shifts, with unknown consequences for plant health and nutrient cycling. Within Australia, altered ECM communities and decreases in ECM colonisation rates have previously been associated with Eucalypt dieback and decreased crown health (Scott et al. 2012; Ishaq et al. 2013; Horton et al. 2013). Additionally, Australia has experienced high rates of mammal extinction and decline (Short and Smith 1994; Woinarski et al. 2015). Combined with our results, these observations raise concerns of major alterations of landscape-level ecosystem function, underscoring the need for further research. Firstly, further studies are needed to confirm whether this ECM community structure is typical for
Australian woodlands. Secondly, future studies should utilise areas where fungal specialists have recently gone extinct or a reduction of mammal diversity has occurred and compare to areas with higher mammal diversity to measure any changes in ECM communities. Thirdly, studies are needed to investigate the functional redundancy of ECM taxa between truffle-like and epigeous taxa for aspects that interact with plant health and nutrient cycling.

Many mycophagy studies have found spores of sporocarpic AM fungi (truffle-like) in mammalian diets, mostly Glomus spp. (Janos et al. 1995; Vernes and Dunn 2009; Nuske et al. 2017). Indeed, these spores have been shown to be viable by inoculating bioassay seedlings with scats containing AM spores (McGee and Baczocha 1994; Reddell et al. 1997). However, our data from an Australian sclerophyll forest show that mammalian diets do not overlap significantly with AM fungi associating with roots or the general soil environment. This indicates that, at least in terms of species diversity, mammal dispersal of AM spores does not have a significant effect on the structure of AM communities in this system. This does not discount other affects mammals may indirectly have on AM communities through physical disturbances or altering plant communities (Gehring and Whitham 1994; Gehring et al.
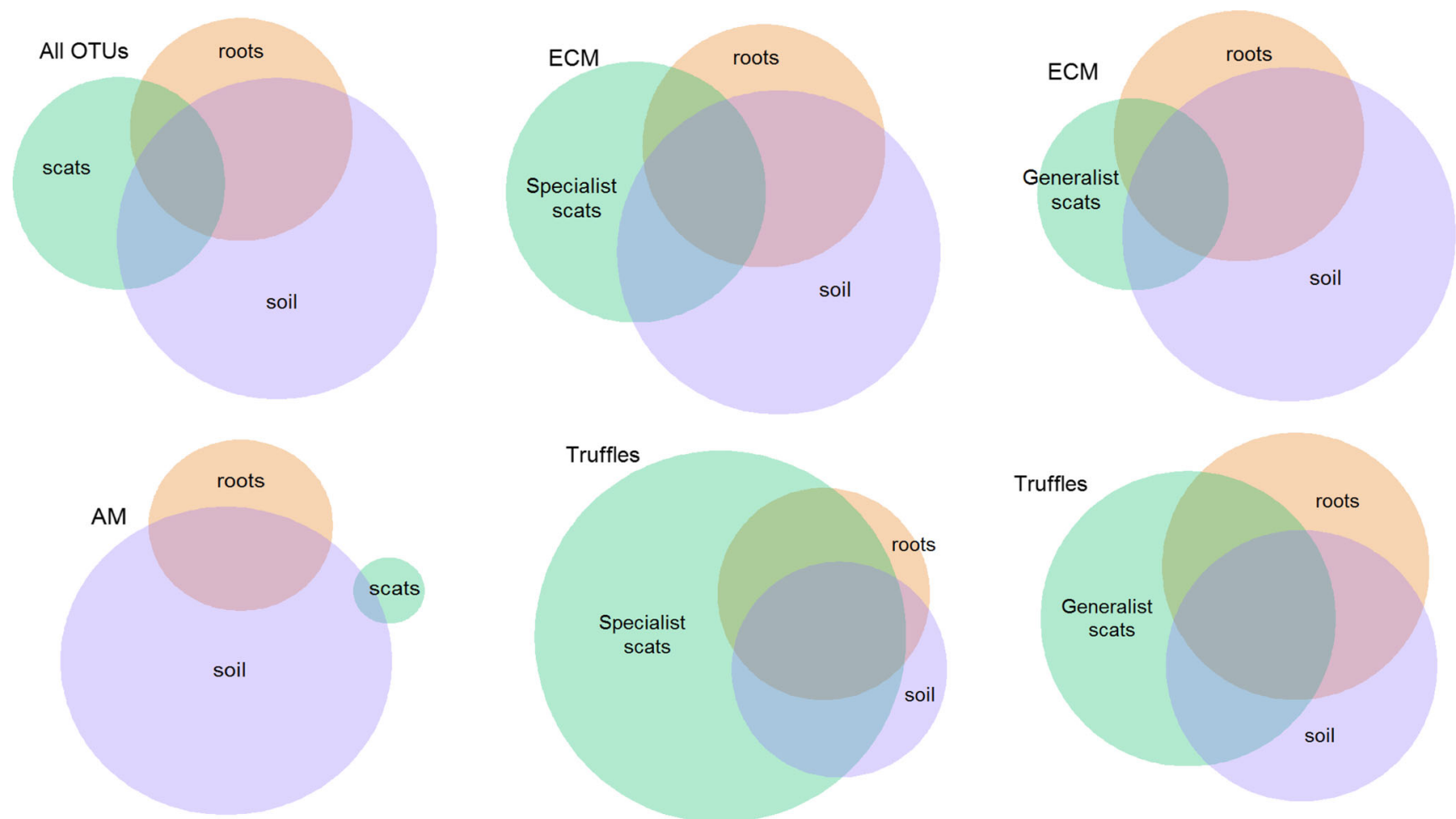

Fig. 4 Venn diagrams; the size of a circle represents the relative OTU richness of each sample type (soil, roots or scats) within each subsample of data (ectomycorrhizal = ECM, arbuscular mycorrhizal = AM, sequestrate/truffle-like $=$ truffles and all OTUs). Overlapping areas represent the proportion of OTUs shared between sample types, whereas the non-overlapping areas represent OTUs unique to specified substrate. The total number of OTUs is 9358,428 for AM fungi, 344 for
ECM fungi and 116 for sequestrate/truffle-like fungi. Specialist scats are from fungal specialist Bettongia tropica, and generalist scats are from Isoodon macrourus, I. obesulus, Melomys sp., Trichosurus vulpecula (ECM only) and Zyzomys argurus. Note: for AM fungi, scats and roots shared $1.8 \%$ of taxa and scats and soil shared $0.8 \%$ of taxa and this is not shown because there were no taxa shared by all samples 

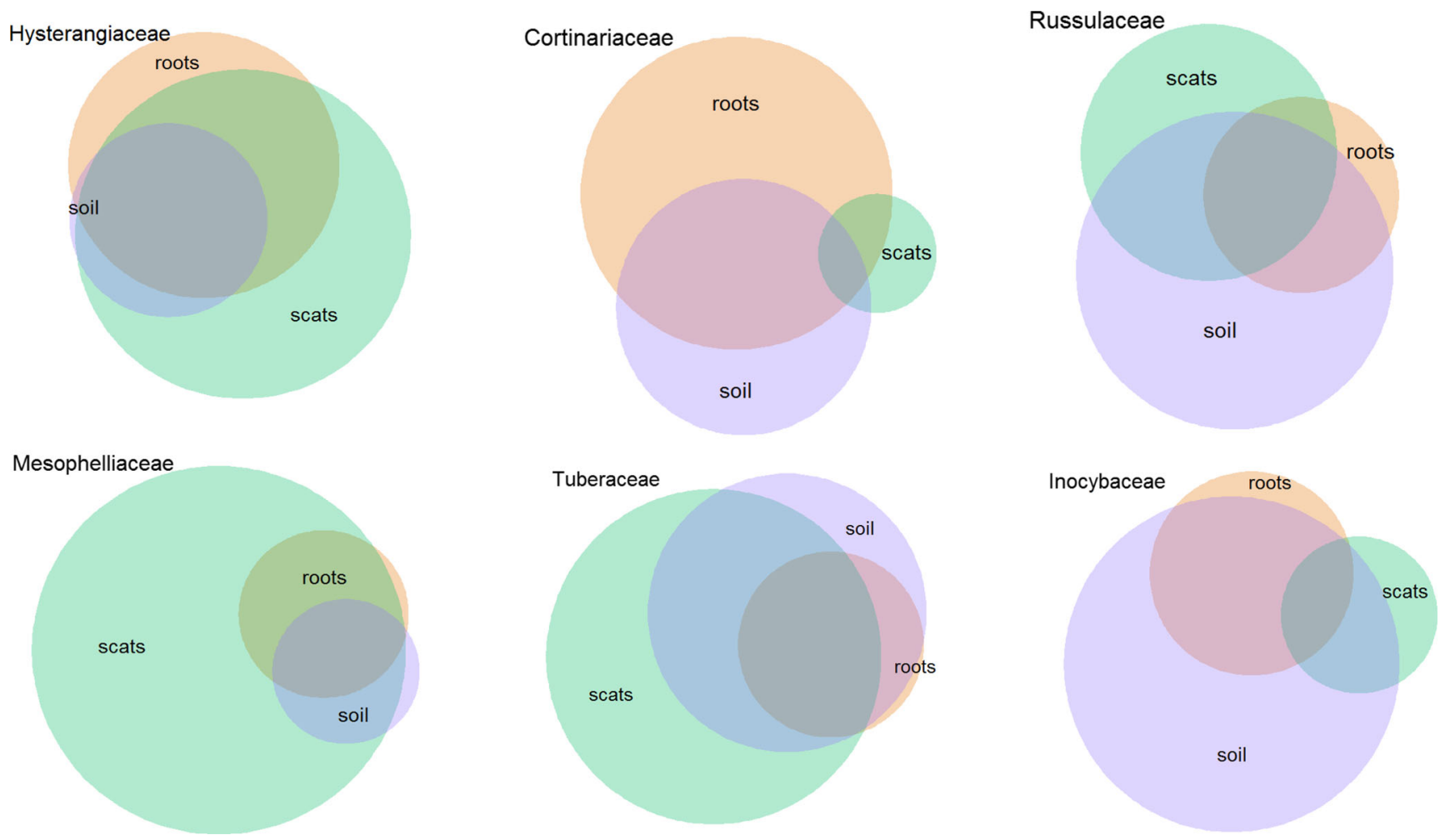

Fig. 5 Venn diagrams; the size of a circle represents the relative OTU richness of each sample type (soil, roots or scats) within each family of fungi (Hysterangiaceae [26 OTUs], Mesophelliaceae [59 OTUs] and Tuberaceae [15 OTUs] contain only truffle and truffle-like/sequestrate species; Cortinariaceae [128 OTUs], Russulaceae [159 OTUs] and
Inocybaceae [132 OTUs] contain truffle-like and/or secotioid species as well as mushroom species). Overlapping areas represent the proportion of OTUs shared between sample types, whereas the non-overlapping areas represent OTUs unique to specified substrate
2002). Other mycophagy studies focusing on AM fungi need to consider the whole community in the soil and on plant roots in order to place appropriate emphasis on these dispersal events for the whole AM fungal community. Nevertheless, mammal dispersal may significantly affect the population structure of sporocarpic AM fungi such as Glomus spp.

Previous ECM community studies in Australia classified between 3 and $27 \%$ of taxa as truffle-like (Online Resource 1, Table S1). We report a percentage of truffle-like taxa within this range $(21 \%)$. We also found that truffle-like taxa comprise dominant portions of the community. Russula and Cortinarius were the most OTU-rich taxa and were included in the relatively abundant groups from our sequencing data and in other ECM surveys in Australia (Online Resource 1, Table S1). However, as many OTUs matching these genera did not match known species, there was not enough taxonomic resolution to discern fruiting habit as these genera contain truffle-like species and mushroom species (e.g. Peintner et al. 2001; Lebel and Tonkin 2007). This limits our capacity to draw conclusions about how truffle-like fungi form part of ECM diversity, and ultimately the overall influence of mycophagous mammals on the ECM community. It also emphasises the need for further targeted truffle-like fungal surveys and taxonomic work on these groups, coupled with continuous updating of online sequence databases.

While we took precautions in this study by removing OTUs present in negative and positive controls, contaminant DNA could still be present and errors in OTU assignment to samples can occur via tag-switching (Carlsen et al. 2012; Nguyen et al. 2015a). For this reason, we consider the proportion of overlapping taxa between sample types to be estimates. Also, taxa we observe at low relative abundances may be indistinguishable from contamination. Amplicon sequencing data are considered 'semi-quantitative' in that relative abundances of sequences within rather than between taxa can be more meaningful as PCR procedures may selectively amplify certain taxa more than others (among other reasons) (Amend et al. 2010). Nevertheless, Nguyen et al. (2015a) argue that relative abundances of taxa may still have ecological value, provided the sequencing errors are appropriately handled and recognised. While we cannot verify whether dominant truffle-like taxa observed were selectively amplified, comparisons within truffle-like taxa (e.g. Hysterangium) show that they have a higher abundance in root-associated communities. OTUs matching truffle-like taxa Malajczukia/Mesophellia, which have a 
high relative abundance in scats, are also present in rootassociating communities. As our results were consistent across two sites and seasons, we consider our assessment of the ECM community structure sufficiently accurate to be confident in the conclusion that truffle-like taxa, and their mammalian dispersers, are important to ECM communities in this system.

Unexpectedly, some OTUs matched truffle sequences of non-Australian taxa (Tuber sp. and Rhizopogon pseudoroseolus). No Tuber species are known to occur natively in Australia, or have associations with native flora (Bonito et al. 2013). Introduced Tuber species associate with introduced trees (mainly on Quercus and Corylus) in temperate regions of Australia (Linde and Selmes 2012; Thomas 2014) and are also known to associate with Pinaceae (Bonito et al. 2013). Rhizopogon species also associate with Pinaceae and other non-native Australian trees (Ivory and Munga 1983; Tedersoo et al. 2007) and have been recorded in Australian pine plantations (Bell and Adams 2004). Incidentally, there is a plantation of Pinus caribaea ca. $10 \mathrm{~km}$ from one of the study sites (Tinaroo Dam) (Applegate and Nicholson 1988). Rhizopogon was found in highest abundance in U. caudimaculatus and Per. nasuta scats at Tinaroo Dam (Nuske et al. 2018); both mammal species have been known to have home ranges within this distance (Scott et al. 1999; Streatfeild 2009). Therefore, it is possible that the OTUs matching R. pseudoroseolus or Tuber sp. resulted from native mammals consuming a non-native, introduced ectomycorrhizae associated with local Pinus plantations. Alternatively, they may have resulted from a contamination from laboratory processing. Nevertheless, the five OTUs identified are unlikely to alter the main findings of this study.

\section{Conclusion}

Diverse ectomycorrhizal fungal communities are vital for healthy ecosystems because of their intimate interactions with plants and pivotal role in nutrient cycling. These fungi also provide food sources for many animals, including the endangered and threatened fungal specialists; species like B. tropica. Little is known about how disturbances to these ecosystems can change ECM fungal communities. Our data show that mammal dispersed truffle-like taxa can form a dominant proportion of root-associating ECM fungal communities. This suggests that changes in the mammal community could, over time, induce changes in functioning ECM fungal communities, which may in turn impact plant health and nutrient cycles on a large scale. Australia has already lost many mammal species and many species are in decline (Woinarski et al. 2015). Conservation of mammal diversity may not only be imperative for ecosystem function at higher trophic levels, but also for maintaining fungal diversity and healthy mycorrhizal-plant relationships.

Acknowledgements Sincere thanks to Queensland Parks and Wildlife Service staff Rob Miller, Lana Little, Jack Cosgrove, Karl Goetze, Chris White, Julie Bunny and Miki Bradley, World Wildlife Fund Australia staff Jessica Koleck, James Cook University students and staff Tegan Whitehead, Stephanie Todd, Naomi Bowie and Elise Chatterton and many field volunteers for help with field work and collection of samples.

Funding sources This research was supported by an Australian Postgraduate Award, Wet Tropics Management Authority (Student Grant 916), Australasian Mycological Society Research Award (2014), North Queensland Wildlife Trust (2014) and Australian Government Caring for our Country 2 Target Area Grant 2013/14 (Project ID: TAG14-00542) Bettongia tropica population status, viability and impact of fire with project partners James Cook University, Queensland Parks and Wildlife Service, Department of Environment and Heritage Protection and World Wildlife Fund Australia (WWF-Australia).

Authors' contributions Nuske, Susan J: designed study; collected data; sequenced DNA; analysed data; wrote manuscript.

Anslan, Sten: preformed bioinformatics; edited manuscript.

Tedersoo, Leho: contributed laboratory space, primers and reagents; provided advice on high-throughput sequencing; edited manuscript.

Congdon, Bradley C: provided advice on design of study and data analysis; edited manuscript.

Abell, Sandra E: provided advice on design of study and data analysis; edited manuscript.

Data availability - Raw Illumina data is deposited in Sequence Read Archive (SRA; bioproject SRP150847).

- Sanger data is available in GenBank (Accessions KY686200KY 686202, KY697566-KY697576, KY697578-KY697619; Online Resource 2).

\section{Compliance with ethical standards}

This research was performed under permits granted by the Queensland Government, EHP (WISP15227914, WITK14639614 and WITK11258712). All wildlife was handled according to JCU Animal Ethics Guidelines (approval number: A2044). The authors declare that they have no conflict of interest.

Open Access This article is distributed under the terms of the Creative Commons Attribution 4.0 International License (http:// creativecommons.org/licenses/by/4.0/), which permits unrestricted use, distribution, and reproduction in any medium, provided you give appropriate credit to the original author(s) and the source, provide a link to the Creative Commons license, and indicate if changes were made.

\section{References}

Abell-Davis SE (2008) Tropical hypogeous fungal sporocarp distribution in time and space. Implications for an endangered specialist mycophagous marsupial, Bettongia tropica. PhD thesis. School of Marine and Tropical Biology. James Cook University

Adams F, Reddell P, Webb M, Shipton W (2006) Arbuscular mycorrhizas and ectomycorrhizas on Eucalyptus grandis (Myrtaceae) trees and 
seedlings in native forests of tropical North-Eastern Australia. Aust J Bot 54:271-281

Amend AS, Seifert KA, Bruns TD (2010) Quantifying microbial communities with 454 pyrosequencing: does read abundance count? Mol Ecol 19:5555-5565. https://doi.org/10.1111/j.1365-294X. 2010.04898.x

Anslan S, Bahram M, Hiiesalu I, Tedersoo T (2017) PipeCraft: flexible open-source toolkit for bioinformatics analysis of custom highthroughput amplicon sequencing data. Mol Ecol Resour May:online first 17:e234-e240. https://doi.org/10.1111/1755-0998.12692

Applegate GB, Nicholson DI (1988) Caribbean pine in an agroforestry system on the Atherton tableland in north East Australia. Agrofor Syst 7:3-15. https://doi.org/10.1007/BF01890466

Bell TL, Adams MA (2004) Ecophysiology of ectomycorrhizal fungi associated with Pinus spp. in low rainfall areas of Western Australia. Plant Ecol 171:35-52. https://doi.org/10.1023/B:VEGE. $0000029372.78102 .9 \mathrm{~d}$

Bonito G, Smith ME, Nowak M, Healy RA, Guevara G, Cázares E, Kinoshita A, Nouhra ER, Domínguez LS, Tedersoo L, Murat C, Wang Y, Moreno BA, Pfister DH, Nara K, Zambonelli A, Trappe JM, Vilgalys R (2013) Historical biogeography and diversification of truffles in the Tuberaceae and their newly identified southern hemisphere sister lineage. PLoS One 8:e52765. https://doi.org/10. 1371/journal.pone.0052765

Bougher NL, Lebel T (2001) Truffle-like (truffle-like) fungi of Australia and New Zealand. Aust Syst Bot 14:439-484

Brundrett MC (2009) Mycorrhizal associations and other means of nutrition of vascular plants: understanding the global diversity of host plants by resolving conflicting information and developing reliable means of diagnosis. Plant Soil 320:37-77

Camacho C, Coulouris G, Avagyan Vet al (2009) BLAST plus: architecture and applications. BMC Bioinformatics 10:421. https://doi.org/ 10.1186/1471-2105-10-421

Carlsen T, Bjørnsgaard Aas A, Lindner D et al (2012) Don't make a mista(g)ke: is tag switching an overlooked source of error in amplicon pyrosequencing studies? Fungal Ecol 5:747-749. https:// doi.org/10.1016/j.funeco.2012.06.003

Claridge AW, May TW (1994) Mycophagy among Australian mammals. Aust J Ecol 19:251-275

Gehring CA, Whitham TG (1994) Interactions between aboveground herbivores and the mycorrhizal mutualists of plants. Trends Ecol Evol 9:251-255

Gehring CA, Wolf JE, Theimer TC (2002) Terrestrial vertebrates promote arbuscular mycorrhizal fungal diversity and inoculum potential in a rain forest soil. Ecol Lett 5:540-548. https://doi.org/10.1046/j.14610248.2002.00353.x

Goto BT, Maia LC (2005) Sporocarpic species of arbuscular mycorrhizal fungi (Glomeromycota), with a new report from Brazil. Acta Bot Bras 19:633-637. https://doi.org/10.1590/S010233062005000300025

Horton BM, Glen M, Davidson NJ, Ratkowsky D, Close DC, Wardlaw TJ, Mohammed C (2013) Temperate eucalypt forest decline is linked to altered ectomycorrhizal communities mediated by soil chemistry. For Ecol Manag 302:329-337. https://doi.org/10.1016/j. foreco.2013.04.006

Ishaq L, Barber PA, Hardy GESJ, Calver M, Dell B (2013) Seedling mycorrhizal type and soil chemistry are related to canopy condition of Eucalyptus gomphocephala. Mycorrhiza 23:359-371. https://doi. org/10.1007/s00572-012-0476-5

Ivory MH, Munga FM (1983) Growth and survival of container-grown Pinus caribaea infected with various ectomycorrhizal fungi. Plant Soil 71:339-344

Izzo AD, Meyer M, Trappe JM (2005) Hypogeous ectomycorrhizal fungal species on roots and in small mammal diet in a mixed-conifer forest. For Sci 51:243-254
Janos DP, Sahley CT, Emmons LH (1995) Rodent dispersal of vesiculararbuscular mycorrhizal fungi in Amazonian Peru. Ecology 76: $1852-1858$

Johnson CN (1996) Interactions between mammals and ectomycorrhizal fungi. Trends Ecol Evol 11:503-507

Lebel T, Tonkin JE (2007) Australasian species of Macowanites are truffle-like species of Russula (Russulaceae, Basidiomycota). Aust Syst Bot 20:355-381. https://doi.org/10.1071/SB07007

Li W, Godzik A (2006) Cd-hit: a fast program for clustering and comparing large sets of protein or nucleotide sequences. Bioinformatics 22: 1658-1659. https://doi.org/10.1093/bioinformatics/btl158

Lindahl BD, Nilsson RH, Tedersoo L, Abarenkov K, Carlsen T, Kjøller R, Kõljalg U, Pennanen T, Rosendahl S, Stenlid J, Kauserud H (2013) Fungal community analysis by high-throughput sequencing of amplified markers-a user's guide. New Phytol 199:288-299

Linde CC, Selmes H (2012) Genetic diversity and mating type distribution of Tuber melanosporum and their significance to truffle cultivation in artificially planted truffiéres in Australia. Appl Environ Microbiol 78:6534-6539. https://doi.org/10.1128/AEM.01558-12

Lu X, Malajczuk N, Brundrett M, Dell B (1999) Fruiting of putative ectomycorrhizal fungi under blue gum (Eucalyptus globulus) plantations of different ages in Western Australia. Mycorrhiza 8:255261. https://doi.org/10.1007/s005720050242

Malajczuk N, Trappe JM, Molina R (1987) Interrelationships among some ectomycorrhizal trees, hypogeous fungi and small mammals: Western Australian and northwestern American parallels. Aust J Ecol 12:53-55

Maser C, Nussbaum RA, Trappe JM (1978) Fungal-small mammal interrelationships with emphasis on Oregon coniferous forests. Ecology 59:799-809. https://doi.org/10.2307/1938784

McGee PA, Baczocha N (1994) Sporocarpic Endogonales and Glomales in the scats of Rattus and Perameles. Mycol Res 98:246-249

McMurdie PJ, Holmes S (2013) Phyloseq: an R package for reproducible interactive analysis and graphics of microbiome census data. PLoS One 8:e61217. https://doi.org/10.1371/journal.pone.0061217

Nguyen NH, Smith D, Peay K, Kennedy P (2015a) Parsing ecological signal from noise in next generation amplicon sequencing. New Phytol 205:1389-1393

Nguyen NH, Song Z, Bates ST, Branco S, Tedersoo L, Menke J, Schilling JS, Kennedy PG (2015b) FUNGuild: an open annotation tool for parsing fungal community datasets by ecological guild. Fungal Ecol 20:1-8. https://doi.org/10.1016/j.funeco.2015.06.006

Nuske SJ, Anslan S, Tedersoo L, Bonner MTL, Congdon BC, Abell SE (2018) The endangered northern bettong, Bettongia tropica, performs a unique and potentially irreplaceable dispersal function for ectomycorrhizal truffle fungi. Mol Ecol 27:4960-4971. https://doi, org $/ 10.1111 / \mathrm{mec} .14916$

Nuske SJ, Vernes K, May TW, Claridge AW, Congdon BC, Krockenberger A, Abell SE (2017) Data on the fungal species consumed by mammal species in Australia. Data Br 12:251-260. https://doi.org/10.1016/j.dib.2017.03.053

Peintner U, Bougher NL, Castellano MA, Moncalvo JM, Moser MM, Trappe JM, Vilgalys R (2001) Multiple origins of truffle-like fungi related to Cortinarius (Cortinariaceae). Am J Bot 88:2168-2179

R Core Team (2012) R: A Language and Environment for Statistical Computing

Reddell P, Gordon V, Hopkins MS (1999) Ectomycorrhizas in Eucalyptus tetrodonta and E. miniata forest communities in tropical northern Australia and their role in the rehabilitation of these forests following mining. Aust J Bot 47:881-907. https://doi.org/10.1071/ bt97126

Reddell P, Spain AV, Hopkins M (1997) Dispersal of spores of mycorrhizal fungi in scats of native mammals in tropical forests of northeastern Australia. Biotropica 29:184-192 
Scott LK, Hume ID, Dickman CR (1999) Ecology and population biology of long-nosed bandicoots (Perameles nasuta) at north head, Sydney harbour National Park. Wildl Res 26:805-821

Scott PM, Shearer BL, Barber PA, Hardy GESJ (2012) Relationships between the crown health, fine root and ectomycorrhizae density of declining Eucalyptus gomphocephala. Australas Plant Pathol 42:121-131. https://doi.org/10.1007/s13313-012-0152-4

Short J, Smith A (1994) Mammal decline and recovery in Australia. J Mammal 75:288-297

Song Z, Schlatter D, Kennedy P, Kinkel LL, Kistler HC, Nguyen N, Bates ST (2015) Effort versus reward: preparing samples for fungal community characterization in high-throughput sequencing surveys of soils. PLoS One 10:e0127234. https://doi.org/10.1371/journal.pone. 0127234

Streatfeild C (2009) The effects of habitat fragmentation on the demography and population genetic structure of Uromys caudimaculatus. $\mathrm{PhD}$ thesis. School of Natural Resource Sciences. Queensland University of Technology

Tedersoo L, Bahram M, Põlme S et al (2014) Global diversity and geography of soil fungi. Science (80-) 346:125668-1-1256688-10. https://doi.org/10.1126/science.aaa1185

Tedersoo L, Suvi T, Beaver K, Kõljalg U (2007) Ectomycorrhizal fungi of the Seychelles: diversity patterns and host shifts from the native Vateriopsis seychellarum (Dipterocarpaceae) and Intsia bijuga (Caesalpiniaceae) to the introduced Eucalyptus robusta
(Myrtaceae), but not Pinus caribea (Pinaceae). New Phytol 175: 321-333. https://doi.org/10.1111/j.1469-8137.2007.02104.x

Thomas PW (2014) An analysis of the climatic parameters needed for Tuber melanosporum cultivation incorporating data from six continents. Mycosphere 5:137-142. https://doi.org/10.5943/mycosphere/5/1/5

Trappe JM, Molina R, Luoma DL, et al (2009) Diversity, ecology, and conservation of truffle Fungi in forests of the Pacific northwest. United States Department of Agriculture, Forest Service, Pacific northwest Research Station. Portland, Oregon

Van Dyck S, Gynther I, Baker A (eds) (2013) Field companion to the mammals of Australia. New Holland Publishers, Sydney

Vernes K (2007) Are diverse mammal communities important for maintaining plant-fungal associations and ecosystem health? Australas Plant Conserv 15:16-18

Vernes K, Dunn L (2009) Mammal mycophagy and fungal spore dispersal across a steep environmental gradient in eastern Australia. Austral Ecol 34:69-76. https://doi.org/10.1111/j.1442-9993.2008.01883.x

Woinarski JCZ, Burbidge AA, Harrison PL (2015) Ongoing unraveling of a continental fauna: decline and extinction of Australian mammals since European settlement. Proc Natl Acad Sci U S A 112: 4531-4540. https://doi.org/10.1073/pnas.1417301112

Publisher's note Springer Nature remains neutral with regard to jurisdictional claims in published maps and institutional affiliations. 